Bio - grafia. Escritos sobre la Biología y su Enseñanza. ISSN 2027

Edición Extraordinaria. p.p. $1460-1470$

Memorias del VIII Encuentro Nacional de Experiencias en Enseñanza de la Biología y la Educación Ambiental. III Congreso Nacional de Investigación en Enseñanza de la Biología.

\title{
Ideas previas sobre el reino vegetal en niños entre los 7 y 10 años de tercero de primaria de la Escuela Normal Superior Distrital María Montessori (E.N.S.D.M.M.)
}

Previous ideas about the plant kingdom in third grade children of the Normal School District Maria Montessori (E.N.S.D.M.M.)

\author{
Gómez L. Daniela \& Velazco T. Daniela ${ }^{1 \star \star}$
}

\section{Resumen}

La presente investigación busca reconocer las ideas previas sobre el reino vegetal en niños entre los 7 y 10 años. Se eligió un grupo de 37 estudiantes de tercero de primaria perteneciente a la E.N.S.D.M.M., con los cuales se implementó un instrumento de caracterización tipo cuestionario conformado por 3 preguntas referentes al reino vegetal. A partir de las respuestas dadas por los niños, se clasificaron sus ideas previas generando categorías de estas, posterior a ello, con la referencia de las 3 fuentes de origen (sensorial, social y analógica) siendo la social en la cual se basa este trabajo; se generaron dos sub-categorias (experiencia cotidiana y experiencia académica) y, en esta última se tuvieron en cuenta los modelos explicativos de nutrición vegetal para lo cual fue necesario analizar conceptos claves como respiración, morfología, metabolismo e interacciones con el medio. Como resultado de la caracterización se determinó que las construcciones mentales sobre el reino vegetal en estos estudiantes son mediadas por la experiencia cotidiana y son frecuentemente nutridas, modificadas o adecuadas por este a pesar de lo adquirido en la escuela, además se estableció que los modelos de nutrición vegetal más comunes en las representaciones de los niños a esta edad son el edáfico y el de transmutación

Palabras Claves: Ideas previas, experiencias cotidianas, reino vegetal.

\section{Abstract}

This research seeks to understand the contributions generated by everruday experiences in the construction of preconceptions about the vegetable in children between 7 and 10 years, to evaluate these contributions a group of 37 third grade students belonging elected to E.N.S.D.M.M., with which caracterization instrument type questionnaire comprised of 3 questions concerning the vegetable was implemented.From the answers given by children rated their preconceptions based on three sources of origin (sensory,

${ }^{1}$ Estudiantes de Licenciatura en Biología. Universidad Distrital Francisco José de Caldas, Facultad de Ciencias y Educación. Contacto ${ }^{+}$daniela.gole@gmail.com ${ }^{++}$dvelascot418@gmail.com 
Bio - grafia. Escritos sobre la Biología y su Enseñanza. ISSN 2027

Edición Extraordinaria. p.p. 1460-1470

Memorias del VIII Encuentro Nacional de Experiencias en Enseñanza de la Biología y la Educación Ambiental. III Congreso Nacional de Investigación en Enseñanza de la Biología.

social and analog) being social in which this paper is based was carried out; likewise also took into account the explanatory models of plant nutrition for which it was necessary to analyze key concepts such as respiration, morphology, metabolism and interactions with the environment. As a result of the characterization was determined that mental constructions of the plant kingdom in these students originate mainly mediated by the social environment and are often nourished, modified or remedies for this, also established that the models most common plant nutrition in representations of children at this age are the edaphic and transmutation strongly influenced by popular knowledge and oral transmission.

Keywords: Previous ideas, everyday experiences, vegetable.

\section{Introducción}

En el proceso de enseñanza de las ciencias naturales siempre surgen explicaciones empíricas por parte de los estudiantes, quienes partiendo de las ideas previas que poseen desarrollan un concepto. Esas ideas son frecuentemente formuladas $y / 0$ influenciadas por espacios sociales y culturales que los rodean, por ende una de las cuestiones que como maestros debemos enfrentar es la existencia de estas, las cuales aparecen como construcciones rígidas presentes a lo largo de la vida escolar, por lo que surge la necesidad de estudiarlas y analizarlas.

Ideas previas: Frente a las ideas previas y la concepción del mundo por parte de los niños varios autores hacen referencia a estas entendiéndolas como promotoras de diferentes escenarios. Según Bello (2004), Flores \& Compañía (1998) y Lacueva \& Rayas (2006), estas se perciben como nociones que poseen los niños sobre diferentes acontecimientos y que no coinciden con el saber científico, siendo fruto de la experiencia directa y cotidiana con objetos y sucesos, de tal manera que son direccionadas por un pensamiento lógico que surge como respuesta a la necesidad de interpretar el entorno. Por otro lado Cubero (1997, pp ), expresa que "son términos, esquemas, representaciones e ideas de los alumnos, (...) las cuales son coherentes internamente y no son al azar. A su vez Driver et al (1999) las asumen como concepciones permanentes que pueden chocar con los resultados esperados por el docente. A su vez Porlan (1993) evidencia que las ideas previas no son fáciles de modificar debido a su coherencia, pues estas se interiorizan es a partir de la experiencia propia. 
Bio - grafia. Escritos sobre la Biología y su Enseñanza. ISSN 2027

Edición Extraordinaria. p.p. 1460-1470

Memorias del VIII Encuentro Nacional de Experiencias en Enseñanza de la Biología y la Educación Ambiental. III Congreso Nacional de Investigación en Enseñanza de la Biología.

Origen de las ideas previa: $\mathrm{Si}$ bien es necesario reconocer las diferentes interpretaciones que se le da a las ideas previas, también se hace fundamental comprender el origen de estas. Cubero (1997) sugiere que al identificar las ideas previa de los estudiantes el docente puede generar un medio de comunicación en el aula y a la vez construir técnicas útiles para explorar y sistematizar lo que piensan los alumnos, y es en este punto donde según Ausubel (1968) citado por García \& Rodríguez (1988) "el factor que más influencia tiene en la enseñanza es lo que el que aprende ya sabe, por lo tanto hay que investigar qué es y enseñar de acuerdo con ello". Rayas.,(s.f) \& Totorikaguena (2013) apuntan a que desde el nacimiento, los niños se ven inmersos en una variedad de fenómenos sociales y naturales, lo cual los lleva a observar y explicar su entorno. Cuadrado (2010) y Cañal (1990) establecen que el origen de las ideas previas en los niños tiene tres fuentes, una sensorial donde hay una concepción espontánea de fenómenos de la vida diaria, una social donde hay concepciones inducidas por las creencias y el entorno, y una de tipo analógico donde se usa un lenguaje cotidiano y ejemplificaciones para la comprensión de fenómenos más complejos. Gómez y Coll (1994) citado por Flores et al (1998) señalan que el conocimiento no es el resultado de una copia de la realidad preexistente sino de un proceso dinámico e interactivo a través del cual la información externa es interpretada y reinterpretada por la mente que va construyendo progresivamente modelos explicativos cada vez más complejos y potentes.

Ideas previas en el contexto social: Retomando a Cuadrado (2010), una de las fuentes de las ideas previas es el medio social, en el cual según Gil (1999), hay una fuerte interferencia del lenguaje científico con el lenguaje cotidiano que tiende a ser imperfecto, Campanario \& Otero (2000) nominan este último lenguaje como el origen de las ideas previas de los estudiantes. Preece (1984) citado por Campanario \& Otero (2000) se refiere a que existe una fuerte influencia de la propia cultura y las creencias en la formación de las ideas previas, y es en esta parte donde el entorno inmediato al niño representa una fuente importante de estímulos para la formulación de ideas previas.

El reino vegetal a través de la historia: El reino vegetal está constituido por todos los organismos clasificados como plantas de las que se sabe que son seres vivos capaces de producir su propio alimento a través de la fotosíntesis (Erice \& Marina, s.f.). Considerando que para elaborar el alimento las plantas desarrollan diversos procesos internos y externos, el hombre a lo largo de la historia ha generado modelos que le permitan comprender la dinámica de estos y otros sistemas vivos. Cañal (1991), Chávez (2002) \& Velázquez (2012) 
Bio - grafia. Escritos sobre la Biología y su Enseñanza. ISSN 2027

Edición Extraordinaria. p.p. 1460-1470

Memorias del VIII Encuentro Nacional de Experiencias en Enseñanza de la Biología y la Educación Ambiental. III Congreso Nacional de Investigación en Enseñanza de la Biología.

concuerdan en mencionar varios modelos explicativos que se han tenido en cuenta desde Aristóteles para explicar la nutrición vegetal entre estos se encuentran: el modelo edáfico que comprende al suelo como alimento de la planta, el de transmutación donde se observa que el agua es la fuente primordial de nutrición de la planta, el flogisto donde ya es considerado el intercambio de gases y el pre científico donde se analizan los procesos bioquímicos que se suceden en la planta.

Contribuciones de otros trabajos en ideas previas sobre el reino vegetal: Contextualizando nuestro trabajo hemos empleado como referentes varios artículos enfocados en el análisis de ideas previas sobre plantas, de estos trabajos rescatamos el de Cañal (1991) que tiene en cuenta concepciones e ideas previas sobre la nutrición en plantas verdes, Velázquez (2011) en el cual se reconocen los modelos explicativos de la nutrición en plantas, Sáenz (2011) tiene en cuenta las concepciones, ideas alternativas y analogías usadas por los estudiantes en fotosíntesis; Tokoricaquena (2013) que aborda los errores conceptuales y las ideas previas del alumnado de ciencias en el ámbito de la enseñanza de la biología celular, y Chávez (2002) que hace un estudio analítico de los modelos explicativos de la nutrición vegetal.

\section{Materiales y Métodos}

A partir de los postulados realizados por Cubero (1997) y Cañal (1991) para explorar y comprender de qué manera el contexto afecta las ideas previas de los estudiantes, se elaboró un instrumento de caracterización tipo cuestionario de carácter abierto evaluado desde el paradigma interpretativo, en el cual se abordaron los reinos de la naturaleza pero, para el presente estudio solo se tuvo en cuenta el reino vegetal, tal elección movilizada tras la reflexión de que las plantas representan una de las primeras aproximaciones de los niños al medio social y cultural.

El instrumento de caracterización se aplicó durante una sesión de dos horas al grado 302 de la (E.N.S.D.M.M.) conformado por 37 estudiantes entre los 7 y 10 años. Se realizaron 3 preguntas relacionadas con el reino vegetal: ¿Reconoces las plantas? ¿Sabes cómo se alimentan? ¿Qué sabes de ellas y cuál crees que sea la importancia de ellas para nosotros y los demás seres vivos?.

Por medio de las ideas descritas por los niños se realizó una clasificación y caracterización de las respuestas a través de la cual se reconocieron y validaron los aportes de la experiencia cotidiana en la construcción de las ideas previas a través de la comparación y análisis de la categoría inducida o social señalada por Cuadrado (2010) y Pozo (1987), se 
Bio - grafia. Escritos sobre la Biología y su Enseñanza. ISSN 2027

Edición Extraordinaria. p.p. 1460-1470

Memorias del VIII Encuentro Nacional de Experiencias en Enseñanza de la Biología y la Educación Ambiental. III Congreso Nacional de Investigación en Enseñanza de la Biología.

establecieron dos sub-categorias, la de experiencia cotidiana y la académica; de esta última se caracterizó el modelo explicativo de nutrición vegetal más constante en los niños por medio de los modelos considerados por Cañal (1990), Cañal (1991), Chávez (2002) \& Velázquez (2012).

\section{Resultados y Discusión}

Gran cantidad de estudios se han venido realizando contextualizados en la enseñanza y el aprendizaje de las ciencias principalmente orientadas a la revisión de las ideas previas. Se hace necesario interpretar estas ideas para entender su origen. Se conformaron 16 categorías, las cuales sub-categorizadas según la incidencia. El $99 \%$ de los estudiantes identifican las plantas aunque algunos las confundan con hongos o algas, también se puede reconocer en las explicaciones dadas por los niños acerca del reino vegetal una construcción inducida basada no solo en experiencias cotidianas, procesos culturales y medios de comunicación (a pesar de ser la más predominante), sino también en experiencias relacionadas con el campo académico (Ver tabla 1).

Tabla 1

Ideas previas de los estudiantes de tercero sobre las características anatómicas, generalidades e importancia de las plantas.

\begin{tabular}{|l|c|l|c|}
\hline $\begin{array}{l}\text { Ideas previas con incidencia de la } \\
\text { experiencia cotidiana }\end{array}$ & $\begin{array}{l}\mathbf{N}^{\circ} \\
\text { de } \\
\text { niños }\end{array}$ & $\begin{array}{l}\text { Ideas previas con incidencia } \\
\text { académica }\end{array}$ & $\begin{array}{l}N^{\circ} \\
\text { de } \\
\text { niños }\end{array}$ \\
\hline $\begin{array}{l}\text { Pueden ser venenosas, toca tener } \\
\text { cuidado con las carnivoras. }\end{array}$ & 1 & $\begin{array}{l}\text { Son verdes, hay de muchos tipos, } \\
\text { tiene pétalos, chuzos, tienen } \\
\text { ramas. }\end{array}$ & 1 \\
\hline $\begin{array}{l}\text { Sirven para hacer remedios o para } \\
\text { que los animales se salven de la } \\
\text { extinción. }\end{array}$ & 2 & $\begin{array}{l}\text { Nacen, crecen, se reproducen y } \\
\text { mueren. }\end{array}$ & 4 \\
\hline $\begin{array}{l}\text { Lo importante es la rosa porque es } \\
\text { como una mujer }\end{array}$ & 1 & $\begin{array}{l}\text { No sólo son importantes para que } \\
\text { respiremos naturalmente sino que } \\
\text { sin ellas moriríamos. Nos dan la } \\
\text { vida }\end{array}$ & 9 \\
\hline
\end{tabular}


Bio - grafia. Escritos sobre la Biología y su Enseñanza. ISSN 2027

Edición Extraordinaria. p.p. 1460-1470

Memorias del VIII Encuentro Nacional de Experiencias en Enseñanza de la Biología y la Educación Ambiental. III Congreso Nacional de Investigación en Enseñanza de la Biología.

\begin{tabular}{|l|c|l|c|}
\hline $\begin{array}{l}\text { Se reproducen por semillas } \\
\text { pequeñas }\end{array}$ & 1 & $\begin{array}{l}\text { Se alimentan de agua y otras cosas } \\
\text { por la raíz. }\end{array}$ & 10 \\
\hline $\begin{array}{l}\text { Se alimentan con cáscaras en una } \\
\text { caja de madera, uno tiene que coger } \\
\text { unos gusanos y meterlos una semana } \\
\text { y ya esta, tienes comida para } \\
\text { plantas }\end{array}$ & 1 & $\begin{array}{l}\text { Se alimentan con el sol y agua, } \\
\text { sustancias nutritivas, tierra, del } \\
\text { aire también, cáscaras }\end{array}$ & 9 \\
\hline $\begin{array}{l}\text { Necesitan cambiar su tierra cada 2 } \\
\text { meses, toman agua, sol, }\end{array}$ & 2 & $\begin{array}{l}\text { Succionan dióxido de carbono y lo } \\
\text { vuelven aire }\end{array}$ & 1 \\
\hline $\begin{array}{l}\text { Si nos les das agua, amor y sol se } \\
\text { mueren }\end{array}$ & 1 & $\begin{array}{l}\text { Hay que cuidarlas con agua, son de } \\
\text { muchos tipos y tienen raíz por } \\
\text { donde se alimentan }\end{array}$ & 2 \\
\hline
\end{tabular}

Se clasifican las ideas previas de los niños según la incidencia social y la incidencia académica, para cada categoría se revisó el $n^{\circ}$ de niños que clasificó con su respuesta en estas sub-categorías.

Para la comprensión de las ideas previas con incidencia de la academia y de la experiencia cotidiana se presenta el análisis basado en los siguientes ítems:

Características anatómicas de las plantas: En primer lugar, se hace evidente que los niños entre los 7 y 10 años generalmente describen las plantas y los demás objetos de su realidad basados en la forma, por ende diferenciar el color, las partes y la forma, son apreciaciones pertinentes para su edad. Se encuentra que los estudiantes reconocen que las plantas toman el alimento del exterior, para así lograr su crecimiento. Se hace evidente que las ideas previas presentadas sobre las plantas están (...) caracterizadas por respuestas rápidas, poco reflexivas, que transmiten mucha seguridad (Gil y Carracosa, 1990, Citados por Tamayo 2002). Un resultado muy similar es el que obtienen Simpson y Arnold (1982) en niños escoceses, cuyas ideas sobre la alimentación de las plantas pueden resumirse en la siguiente: las plantas consiguen su comida del suelo, por las raíces. Partiendo de dichas ideas, introducimos la existencia y la permanencia de éstas (Totorikaguena, 2013) con la condición repetitiva de experiencias siendo el producto de un extenso proceso de aprendizaje basado en experiencias diarias. 
Bio - grafia. Escritos sobre la Biología y su Enseñanza. ISSN 2027

Edición Extraordinaria. p.p. 1460-1470

Memorias del VIII Encuentro Nacional de Experiencias en Enseñanza de la Biología y la Educación Ambiental. III Congreso Nacional de Investigación en Enseñanza de la Biología.

Fuente de alimentos y su importancia para otros seres vivos: Analizando las respuestas dadas por lo niños, se ve reflejada la conexión que hacen ellos entre la planta y el medio, pues señalan una relación entre el agua, la tierra, la raíz y el sol; algunos hablan de las cáscaras como alimento, lo que concuerda con las conclusiones a las que llegaron Charrier M. y Compañia (2006) citados por Velazquez (2011), \& Cañal (1991) de que gran parte de los estudiantes, sobre todo los más pequeños, piensan que las plantas obtienen todo su alimento del suelo, por medio de las raíces. Cesalpino (1584) recalca que "en su época se mantiene laidea de que la planta toma los materiales nutritivos del suelo, por las raíces, siendo conducidos por las venas hasta el tallo y de ahí a los frutos, (su producción se considera que es lo que da sentido a este tipo de ser vivo); con lo anterior que se resalte un gran paso, pues los estudiantes desde su experiencia reconocen la importancia de las plantas como productoras y las sitúan como parte fundamental de un ecosistema. Como bien lo menciona (Roth, Smith y Anderson, 1983) citados por Velázquez (2011) los niños casi siempre desconocen la importancia de la luz para las plantas, afirmación evidente en las respuestas de estos estudiantes. Velázquez (2011), respalda que los estudiantes consideran que las plantas utilizan la energía solar para mantenerse saludables, a lo que el actual trabajo ve latente en las ideas previas de los niños.

Producción de oxígeno: "Nos dan oxígeno y la vida" señalan varios estudiantes, quienes reconocen que las plantas emplean el aire de forma contraria que los animales, Giordan (1990) cita que más del $80 \%$ de los alumnos de 10 a 12 años ha oído hablar del desprendimiento de oxígeno por las plantas, igualmente consideran necesaria la luz para éstas. Cañal (1991) predice que el proceso de respiración de las plantas no es algo obvio, provisto de signos externos fácilmente observables como en el caso de los animales, pero sin embargo es claro que más del $80 \%$ de los estudiantes comprendidos entre los 7 y los 10 años que contestaron la encuesta, reconocen dicho proceso, pero sin lograr explicarlo. Dentro de los cuatro niveles de construcción que establece Cañal en 1990, en escolares de 9 a 13 años encontramos la presencia de ideas en el campo conceptual de la respiración en el primer nivel, siendo éste en el cual no se logra apreciar una "definición personal sobre alimentación, la fotosíntesis y la respiración de las plantas. Tan sólo se aprecia una cierta proximidad hacia la concepción de la alimentación como proceso externo y simple: captación de sustancias del suelo".

Modelos explicativos de la nutrición en plantas.: Los estudiantes han elaborado diferentes modelos para explicarse los procesos de alimentación en las plantas, muchas de las concepciones son producidas a partir de la experiencia y del conocimiento desarrollado en la escuela, otras simplemente son producto de la interacción con el entorno social en el 
Bio - grafia. Escritos sobre la Biología y su Enseñanza. ISSN 2027

Edición Extraordinaria. p.p. 1460-1470

Memorias del VIII Encuentro Nacional de Experiencias en Enseñanza de la Biología y la Educación Ambiental. III Congreso Nacional de Investigación en Enseñanza de la Biología.

que se encuentran activos y otras son producto del debate que según Reyzabal (1994) es por medio de esta herramienta de transmisión oral que se hace un intercambio de opiniones que infiere en las ideas del otro.

Se hace explícito que las respuestas dadas por los alumnos principalmente corresponden al modelo explicativo edáfico mencionado por Velázquez (2011) cuya principal premisa asume a las raíces como boca de las plantas y al suelo como componente nutricio de la planta Hay nociones de los modelos Transmutacionales y Flogistos cuando los niños consideran el agua como mediadora de los procesos hechos por la planta y al intercambio gaseoso como motor del funcionamiento de la misma, más no de modelos pre científicos que consideran ya procesos bioquímicos y en orgánulos específicos de los órganos de la planta. Ahora partiendo de la clasificación dada por Cañal (1990) tenemos que los niños valorados en este estudio se encuentran en los dos primero niveles, pues en el tercer y cuarto nivel ya se generan relaciones entre conceptos de fotosíntesis, absorción, respiración y procesos bioquímicos internos.

Tradiciones culturales, creencias y medios de comunicación: Para el caso de los niños de grado 3 de la E.N.S.D.M.M. se evidencia una manifestación de ideas previas inducidas por su contexto a pesar de que el docente presenta conceptos con argumentos científicos, estas se manifiestan principalmente quizás obedeciendo a la baja articulación curricular como lo expresa Saenz (2012). La constancia de las ideas previas está directamente atada a la repetición de las experiencias, y de alguna manera si estas experiencias están atadas al contexto, se verán más fuertemente relacionadas por el niño. Totorikaguena (2013). También se logra ver cierta construcción de estas ideas en la experiencia al expresar su función a nivel medicinal, que cuando se marchitan mueren, su relación con la tierra y el agua, así como la producción de oxígeno y su importancia para la respiración y la vida del hombre y demás seres vivos. Las transformaciones en los modos como circula el saber constituye una de las más profundas transformaciones que una sociedad puede sufrir (Barbero, 2002). "Se alimentan con cáscaras en una caja de madera; uno tiene que coger unos gusanos y meterlos una semana y ya está tienes comida para las plantas" esta respuesta nos dispone a señalar que existe una tendencia por considerar que las plantas toman su medio del exterior, por medio de las raíces; dicha afirmación en base a experiencias que los estudiantes tienen como el compostaje o el uso frecuente de plantas como medicinas. La trasmisión oral, la cultura y las costumbres son fundamentales, pero también los recursos audiovisuales, la televisión y el internet cobran un papel muy importante en la generación de estas concepciones en los niños. Las relaciones psicosociales 
Bio - grafia. Escritos sobre la Biología y su Enseñanza. ISSN 2027

Edición Extraordinaria. p.p. 1460-1470

Memorias del VIII Encuentro Nacional de Experiencias en Enseñanza de la Biología y la Educación Ambiental. III Congreso Nacional de Investigación en Enseñanza de la Biología.

y afectivas promueven muchas de las respuestas dadas por los estudiantes, tales como "lo importante de la flor es la rosa porque es como una mujer".

\section{Conclusiones}

Las construcciones mentales de los estudiantes y sus ideas previas sobre el reino vegetal y la nutrición de las plantas están fuertemente influenciadas por la experiencia, la tradición oral, y el contexto social en el que se desarrollan, aunque la incidencia académica se ve reflejada en muchas de las ideas expresadas, no se logra observar una apropiación por parte de los estudiantes.

Los estudiantes que participaron en este estudio tienen ideas previas sobre las plantas similares a las expresadas en otros estudios, por lo que se hace evidente que las ideas previas pueden ser mediadas por las edades.

Por medio de la revisión de las categorías establecidas para los modelos de nutrición vegetal que se manifiestan en estudiantes, se determina que la gran mayoría, explican la nutrición vegetal desde el modelo edáfico y el modelo de transmutación, donde se reconoce principalmente la relación entre la planta, con la tierra, la raíz y el agua.

\section{Referencias}

Ausubel, D. (1968) Educational Psichology: A cognitive view. (Rinehart y Winston. New York).

Barbero, J.M. (2002). La educación desde la comunicación. III Reconfiguraciones comunicativas del saber y del narrar. Tecnologías de Información y Comunicaciones para enseñanza Básica y Media.

Barbero, J.(2002). La educación desde la comunicación. Reconfiguraciones comunicativas del saber y del narrar. Tecnologías de Información y Comunicaciones para enseñanza Básica y Media.

Campanario, J.M. Otero, J.C. (2000). Más allá de las ideas previas como dificultades de aprendizaje: las pautas de pensamiento, las concepciones epistemológicas y las estrategias metacognitivas de los alumnos de ciencias. Universidad de Alcalá. Madrid. 
Bio - grafia. Escritos sobre la Biología y su Enseñanza. ISSN 2027

Edición Extraordinaria. p.p. 1460-1470

Memorias del VIII Encuentro Nacional de Experiencias en Enseñanza de la Biología y la Educación Ambiental. III Congreso Nacional de Investigación en Enseñanza de la Biología.

Cañal, P. (1990). La enseñanza en el campo conceptual de la nutrición de las plantas verdes. Un estudio didáctico en la educación básica. Tesis doctoral inédita. Universidad de sevilla. Cañal, P. (1990). La enseñanza en el campo conceptual de la nutrición de las plantas verdes. Un estudio didáctico en la educación básica. Tesis doctoral inédita. Universidad de sevilla.

Cañal, P. (1991). Las concepciones sobre la nutrición de las plantas verdes. Dpto. de Didáctica de las Ciencias. Universidad de sevilla. pp. 8- 27.

Cañal, P. (1991). Las concepciones sobre la nutrición de las plantas verdes. Dpto. de Didáctica de las Ciencias. Universidad de sevilla. pp. 8- 27.

Chavez, M. (2002). Estudio analítico no lineal de los modelos explicativos de la nutrición vegetal y su valor para el proceso de enseñanza- aprendizaje. Revista TED: Tecne, Episteme y Didaxis, $N^{\circ} 11$, universidad pedagógica de Colombia, pp. 3-14.

Cuadrado, M, F. (2010). Los conocimientos previos que poseen el alumnado de educación primaria sobre ciencias y como aprenden. Didácitca. Innovación y experiencias educativas, pp. 2-7.

Cubero, R. (1997). Como trabajar con las ideas de los alumnos. DIADA, pp 42-46.

Gil, P.D. y Carracosa, J. (1990). What to do about science "misconceptions", Science Education, pp. 531-540

Giordan, A. (1990). Interests des recherdies en didactique de la biologie. LDES. Université de Genevé.

Miller, K, R. Levine, J. (2004). Biología. Prentice Hall. Pearson, pp. 578-589.

Porlán, A, R. García, R.A. Del Pozo, M. (1997). Conocimiento profesional y epistemología de los profesores I: teorís, métodos e instrumentos.

Pozo, J, I. (1987). Y sin embargo se puede enseñar ciencias. Universidad autónoma de Madrid. Infancia y aprendizaje, pp. 109- 113. 
Bio - grafia. Escritos sobre la Biología y su Enseñanza. ISSN 2027

Edición Extraordinaria. p.p. 1460-1470

Memorias del VIII Encuentro Nacional de Experiencias en Enseñanza de la Biología y la Educación Ambiental. III Congreso Nacional de Investigación en Enseñanza de la Biología.

Preece, P. (1984) intuitive science:learned or tiggered. European Journal of science Education, 6, pp. 7-10.

Reyzabal, M. V. (1994): La lírica. Técnica de comprensión y expresión. Madrid: Arco/Libros

Saenz G. J. (2012) La fotosintesis, concepciones, ideas alternativas y analogías. Unidad didáctica dirigida a estudiantes de los ciclos 3 y 4 de educación básica del colegio José María Carbonell .Universidad Nacional de Colombia Facultad de Ciencias Bogotá, Colombia.

Simpson, M. Y Arnold, B. (1982). Avaliability of prerequisite concepts for learning biology at certificate level. Journal of biological education. 16(1), pp. 65-72.

Tamayo, A. O. (2002). De las concepciones alternativas al cambio conceptual. Plumilla Educativa, pp. $57-65$

Totorikaguena, L.I. (2013) Los errores conceptuales y las ideas previas del alumnado de ciencias en el ámbito de la enseñanza de la biología celular. Propuestas alternativas para el cambio conceptual. Leioa, Universidad del país Vasco, pp. 9- 14.

Velázquez, O. (2011) Modelos explicativos sobre el concepto de nutrición en plantas en estudiantes de básica secundaria rural, Universidad Nacional de Colombia Facultad de Ciencias Exactas y Naturales Maestría en Enseñanza de las Ciencias Exactas y Naturales Manizales. 